\title{
Podoplanin is an efficient predictor of neck lymph node metastasis in tongue squamous cell carcinoma with low tumor budding grade
}

\author{
MEI HAMADA ${ }^{1,2}$, YASUHIRO EBIHARA ${ }^{3}$, KOJI NAGATA $^{1}$, MITSUTAKE YANO $^{1,4}$, YASUNAO KOGASHIWA ${ }^{3}$, \\ MITSUHIKO NAKAHIRA ${ }^{3}$, MASASHI SUGASAWA $^{3}$, HITOSHI NAGATSUKA $^{2}$ and MASANORI YASUDA ${ }^{1}$ \\ ${ }^{1}$ Department of Pathology, Saitama Medical University International Medical Center, Hidaka, Saitama 350-1298; \\ ${ }^{2}$ Department of Oral Pathology and Medicine, Graduate School of Medicine, Dentistry and Pharmaceutical Sciences, \\ Okayama University, Okayama 700-8525; ${ }^{3}$ Department of Head and Neck Surgery, Saitama Medical University \\ International Medical Center, Hidaka, Saitama 350-1298; ${ }^{4}$ Department of Obstetrics and Gynecology, \\ Oita University Faculty of Medicine, Oita 879-5593, Japan
}

Received October 1, 2019; Accepted December 31, 2019

DOI: $10.3892 / \mathrm{ol} .2020 .11358$

\begin{abstract}
The current study investigated the efficacy of podoplanin expression in tumor budding cells as a predictor of neck lymph node metastasis (NLM) in patients with tongue squamous cell carcinoma (SCC) of low tumor budding grade (TBG). A total of 99 patients with early T-stage tongue SCC of any clinical $\mathrm{N}$ status who received the initial curative treatment were enrolled. The association between podoplanin expression and NLM was immunohistochemically analyzed, with a focus on tongue SCC with low TBG. The disease-specific survival (DSS) rate was $77 \%$ at 5 years, and a significant difference was observed between the NLM-positive and NLM-negative groups, and between the low $(n=77)$ and high $(n=22)$ TBG groups. In the low TBG group, there was a significant difference in DSS between the NLM-positive and NLM-negative groups. The multivariate analysis showed that lymphatic vessel invasion (ly) [odds ratio $(\mathrm{OR})=11.5,95 \%$ confidence interval $(\mathrm{CI})$ : $1.50-87.6 ; \mathrm{P}=0.02]$ and podoplanin expression $(\mathrm{OR}=7.07,95 \% \mathrm{CI}: 1.80-27.7 ; \mathrm{P}=0.005)$ were significantly correlated with NLM. Furthermore, negative predictive values (NPV) of ly and podoplanin expression for NLM were $75 \%$ and $88 \%$, respectively. Considering the balance of stratification case number adding to ratio, NLM-negative prediction by podoplanin was more significant than that by ly for the low
\end{abstract}

Correspondence to: Dr Masanori Yasuda, Department of Pathology, Saitama Medical University International Medical Center, 1397-1 Yamane, Hidaka, Saitama 350-1298, Japan

E-mail:m_yasuda@saitama-med.ac.jp

Abbreviations: DOI, depth of invasion; DSS, disease-specific survival; EMT, epithelial mesenchymal transformation; ly, lymphatic vessel invasion; NLM, neck lymph node metastasis; NPV, negative predictive values; pDiameter, pathological diameter of tumor; TBG, tumor budding grade; $\mathrm{v}$, vascular vessel invasion

Key words: neck lymph node metastasis, podoplanin expression, squamous cell carcinoma, tongue, tumor budding grade
TBG group. The results of the present study demonstrated that podoplanin expression in tumor budding is an independent and efficient predictor of NLM in the tongue SCC with low TBG. The low TBG and podoplanin-negative cases may be candidates for the wait and watch policy, therefore, reducing inappropriate elective neck lymph node dissections.

\section{Introduction}

The tongue squamous cell carcinoma (SCC) is the most common oral cancer, and even in the early stage, some cases may be aggressive with poor prognosis. Therefore, the National Comprehensive Center Network guideline (1) recommends elective neck lymph node dissection even for clinical N0 cases when the depth of invasion (DOI) of the tumor is greater than $4 \mathrm{~mm}$. However, more than half of the patients undergo inappropriate neck dissections (2), and therefore, establishment of a proper neck lymph node metastasis (NLM) predictor-that can be determined from the primary lesion-has long been sought. Clinical N status determination includes various bias related to the influence of the modality and evaluator. In addition, cervical lymph node metastasis cannot be assessed at the cellular level with images. In contrast, pathological results provide a more precise assessment of lymph node metastasis with little bias, regardless of clinical $\mathrm{N}$ status.

Studies have shown that tumor thickness $(3,4)$, DOI $(5,6)$, lymphatic vessel invasion (ly) or vascular vessel invasion (v) (7), perineural invasion (8), worst pattern of invasion (9), and YK classification (10) are pathological factors that correlate with NLM. Among these, DOI has been adopted in the recent UICC TNM classification (11), but there is no clear threshold for predicting NLM, which definitely distinguishes NLM presence from absence for reliable practical use.

Recently, tumor budding has been drawing considerable attention. A tumor budding nest, which consists of a single or less than five cancer cells present on the invasive front of cancer, closely correlates with NLM and prognosis of colon and other cancers, and tumor budding grade (TBG) has been incorporated into the treatment algorithm of colon cancer (12). Some previous studies of SCC in head and neck have shown 
the correlation of NLM with TBG, but they were limited to high TBG cases $(13,14)$. In our previous study $(15)$, we found that TBG is the most important prognostic factor for the early T-stage tongue SCC. High TBG indicates a high risk for NLM, and is superior to DOI at predicting NLM. The majority of patients with high TBG showed NLM, and minority of those with low TBG had NLM. However, in terms of the total number of patients with NLM, they were almost evenly divided into low and high TBG groups, indicating that the number of NLM-positive patients in the low TBG group could not be ignored. Hence, there is a demand for an NLM predictor that can be combined with TBG for predicting tongue SCC with low TBG.

Podoplanin has long been known to be a lymphatic endothelial marker, but has recently been reported to be expressed in a variety of tumors including SCC (16). In addition, it is a marker of stem cell, and is associated with tumor lymphangiogenesis and promotes metastasis by aggregating platelets, consequently preventing immune attack. It is noteworthy that podoplanin expressed cells have been reported to be located at the invasive front of tumors, to have a high metastatic potential $(17,18)$, and podoplanin expression in dysplastic epithelium could be a predictive marker for tumorigenesis in precancerous lesions (19). The expression of podoplanin at the front of a tumor invasion is observed in routine diagnosis, but there is no report examining the significance of podoplanin expression in tumor budding.

The purpose of this study was to investigate whether podoplanin expression in tumor budding in tongue SCC with low TBG might be an efficient NLM predictor. This is the routine-based method of extracting cases with low TGB, but which may potentially have a high NLM risk. This may contribute to prognosis prediction and treatment planning for tongue SCC patients.

\section{Materials and methods}

Patients and samples. In this retrospective study, 99 patients with tongue SCC at clinically early T-stages of any clinical N status according to the UICC TNM seventh edition (20), who underwent surgical resection in the Saitama Medical University International Medical Center between 2007 and 2016, were enrolled. These patients had to meet the following criteria: no previous history of neoadjuvant therapy, a minimum follow-up period of 1 year for surviving cases, adequate specimens for histological observation and immunohistochemical analysis, and histologically confirmed invasion. The pathological stage was determined according to the UICC TNM eighth revised edition (11), and the cut off values for DOI and thickness were also based on the classification.

The Institutional Review Board at the Saitama Medical University International Medical Center approved this study (approval no. 17-201). All methods were performed in accordance with the 1975 Declaration of Helsinki.

Histopathological evaluation of TBG. According to the previous study $(21,22)$, specimens were scanned to determine the area with the highest density of budding and observed under a x20 objective lens and a x10 ocular lens. TBG was classified as 1-3 according to the number of tumor buds: 1,0 to 4 buds; 2, 5 to 9 buds; and 3, $\geq 10$ buds (Fig. 1). Two authors (HM and NK) independently evaluated each case according to the criteria mentioned above using hematoxylin-eosin-stained and cytokeratin AE1/3-stained slides. Disagreements between the two assessors were resolved by re-reviewing them or having them reviewed by another assessor (YM). Cytokeratin AE1/3-staining was useful for a less experienced pathologist (HM), as previously reported (23). The cases were divided into two groups: high TBG (TBG3) and low TBG (TBG1/2) based on previous studies $(14,15)$.

Immunohistochemistry and evaluation. Paraffin sections of 4- $\mu \mathrm{m}$ thickness were immunostained with the AE1/3 antibody (PCK26, cocktail antibody, Ventana; Roche Tissue Diagnostics Japan; cat. no. 760-2595) and the podoplanin antibody (clone D2-40, Dako Agilent Technologies, Inc.; cat. no. M3619), respectively, using an automated immunostainer (VENTANA BenchMark ULTRA system; Ventana Medical Systems, Inc.) according to the manufacturers' protocol. The incubation with secondary antibodies and detection were carried out using the I-VIEW DAB Universal kit (Ventana Medical Systems, Inc.; cat. no. 760-041) and Endogenous Biotin Blocking kit (Ventana Medical Systems, Inc.; cat. no. 760-050).

Podoplanin expression in budding cells, which was confirmed by AE1/3 expression, was evaluated independently by the same authors mentioned above (HM and NK). The results were scored from 0 to 3 based on the intensity of the staining at the membrane or in the cytoplasm: 0 , no reactivity; +1 , weak; +2 , moderate; and 3, marked, that is, overexpression (Fig. 2). For analysis, the results were divided into 2 groups: negative (score 0 ) and positive (from +1 to +3 ). The ly of the tumor tissue was evaluated using hematoxylin and eosin staining or podoplanin staining for detecting lymphatic vessels (negative; positive).

Statistical analysis. The clinicopathological characteristics of the patients were compared using the Chi-square test or Fisher's exact test. Disease-specific survival (DSS) rate was compared using Kaplan-Meier method with log-rank test and Cox proportional hazards model for a multivariate analysis. The independent prognostic strength of NLM was determined using a logistic regression model. All statistical analyses were performed using IBM SPSS software (version 24.0; IBM Corp.), and $\mathrm{P}<0.05$ was considered to indicate a statistically significant difference.

\section{Results}

The 99 patients comprised 72 males and 27 females with a median age of 63 years old (range: $20-89$ years) and median follow-up of 39 months (range: 6-121 months) (Table I). Forty-three patients were categorized as wait and watch and neck dissection was performed for 56 patients. After primary resection, 14 patients without neck dissection were classified as doubtful NLM and subsequently subjected to therapeutic neck dissection. Pathological lymph node metastasis was present in 39 cases consisting of 27 cases with initial neck dissection and 12 cases with therapeutic neck dissection. In clinical N0 patients, there was no difference in the disease specific survival rate between patients in the wait and watch 

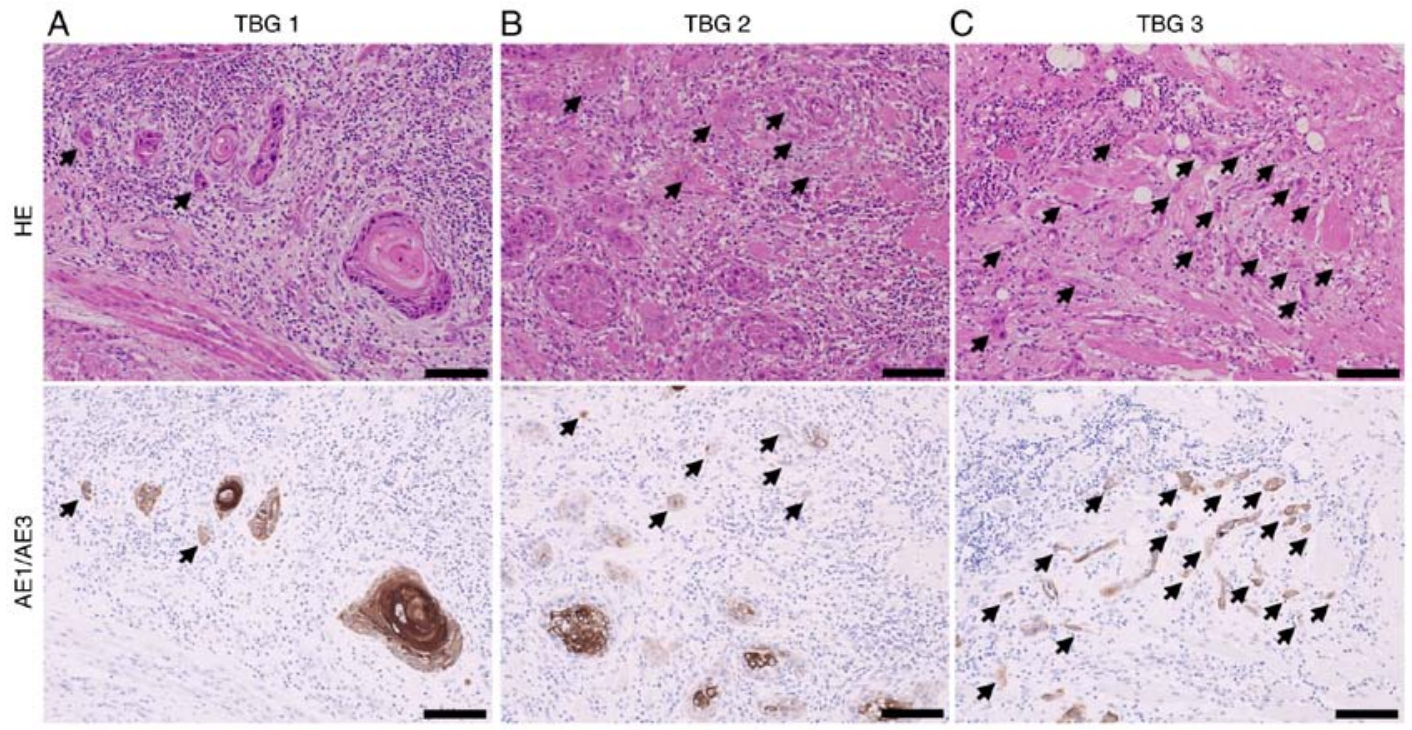

Figure 1. Immunohistochemical analysis for TBG by Pan-Cytokeratin AE1/3 staining. Budding is recognized as a single cancer cell or cancer clusters consisting of four cells or less at the invasive front shown by the arrows. (A) TBG 1: budding counts 0-4. (B) TBG 2: budding counts 5-9. (C) TBG 3: budding counts more than nine. Each field is magnification, 20x10. Scale bar, $100 \mu \mathrm{m}$. TBG, tumor budding grade; HE, hematoxylin and eosin.

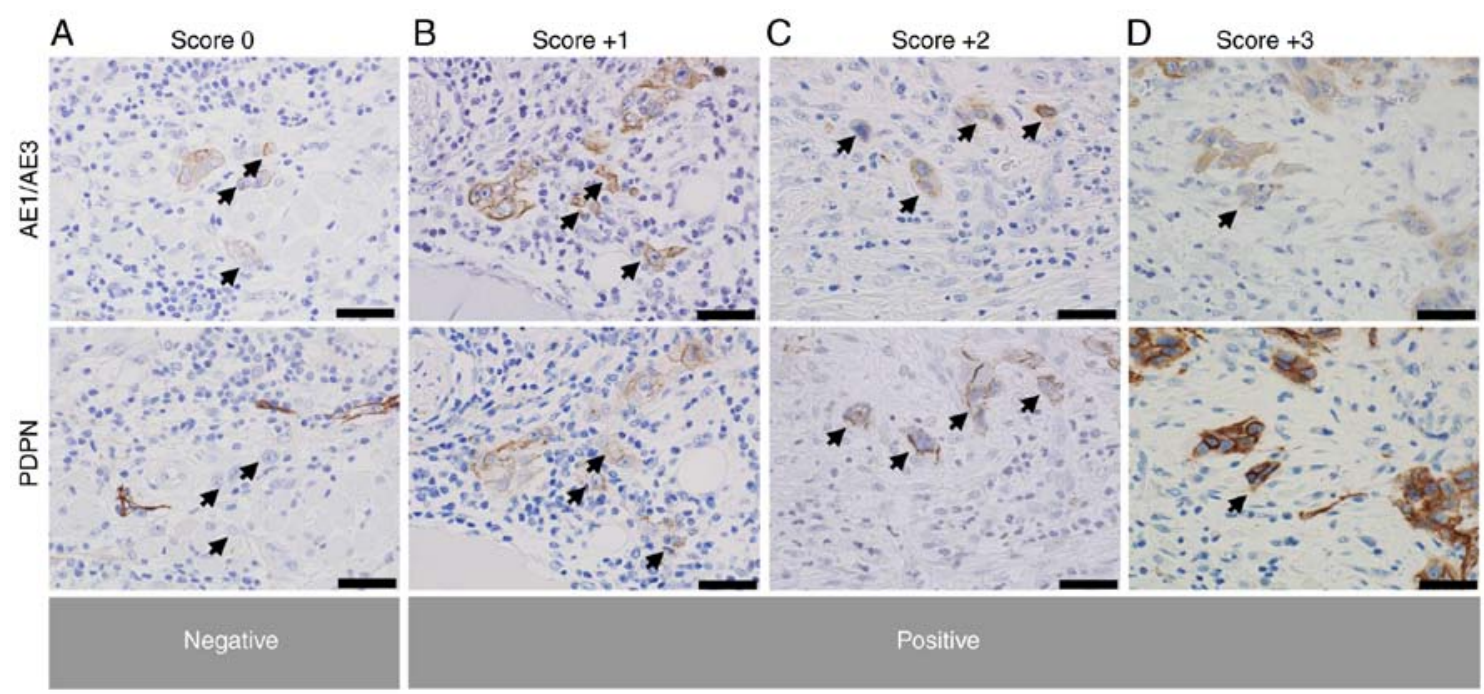

Figure 2. Scoring of PDPN in tumor budding cells by immunohistochemical stain analysis with D2-40. After confirming budding cells by Pan-Cytokeratin AE1/3 staining (upper figures), the PDPN score was judged in the same budding cells (lower figures). (A) was judged to be score 0 and (B), (C), and (D) were judged to be scores 1,2, and 3, respectively. For statistical analysis (A) is determined to be negative. (B), (C), and (D) are determined to be positive. The arrows indicate each bud and each field at magnification, 60x10. Scale bar, $40 \mu \mathrm{m}$. PDPN, podoplanin.

group and the elective neck dissection group $(\mathrm{P}=0.883)$. At that time, elective neck dissection was recommended for patients with late $\mathrm{T} 2$ (diameter of $\geq 30 \mathrm{~mm}$ ) tumors. In regard to intermediate tumors that were between superficial tumors and late $\mathrm{T} 2$ tumors, physicians individually considered tumor thickness, localization, and the patient's health condition.

TBG evaluation resulted in 41 patients (41\%) for TBG1, 36 (36\%) for TBG2, and 22 (22\%) for TBG3. Based on podoplanin expression, 39 patients (39\%) were negative and 60 patients $(61 \%)$ were positive: weak $(+1), 35$ patients; moderate $(+2)$, 14 patients; marked ( +3$), 11$ patients. The final outcome was survival in 76 patients and death in 23 patients: disease-specific death, 17 patients; other causes of death, 6 patients (1 heart disease-related death, 2 had carcinomas apart from the head and neck, and 3 had pneumonia). DSS rate was $77 \%$ at 5 years (Fig. 3A).

In the low TBG group, there were significant differences in the DSS between the NLM-positive and NLM-negative groups $(\mathrm{P}=0.010$; Fig. 3B) and between the podoplanin-positive and podoplanin-negative groups $(\mathrm{P}=0.006)$. In the low $\mathrm{TBG}$ with clinical N0 group ( $\mathrm{n}=53)$, there was no difference of disease specific survival rate between patient with wait and watch group $(n=36)$ and with elective neck dissection group $(n=17)$ ( $\mathrm{P}=0.803$; Fig. 3C).

Correlations between NLM and variable clinicopathological parameters such as $\mathrm{pT}(\mathrm{P}=0.004)$, pathological diameter (pDiameter) $(\mathrm{P}=0.012)$, DOI $(\mathrm{P}=0.024)$, ly $(\mathrm{P}=0.001)$, $\mathrm{v}(\mathrm{P}=0.001)$, TBG $(\mathrm{P}<0.001)$, and podoplanin $(\mathrm{P}=0.007)$ were 
Table I. Patient characteristics and NLM analysis.

\begin{tabular}{|c|c|c|c|c|c|c|c|c|}
\hline \multirow[b]{3}{*}{ Characteristics } & \multicolumn{4}{|c|}{ All cases $(n=99)$} & \multicolumn{4}{|c|}{ Low TBG cases $(n=77)$} \\
\hline & \multirow[b]{2}{*}{ No. } & \multicolumn{2}{|c|}{ NLM } & \multirow[b]{2}{*}{ P-value ${ }^{a}$} & \multirow[b]{2}{*}{ No. } & \multicolumn{2}{|c|}{ NLM } & \multirow[b]{2}{*}{ P-value ${ }^{a}$} \\
\hline & & $\begin{array}{c}-(\%) \\
60(61)\end{array}$ & $\begin{array}{c}+(\%) \\
39(39)\end{array}$ & & & $\begin{array}{c}-(\%) \\
54(70)\end{array}$ & $\begin{array}{c}+(\%) \\
23(30)\end{array}$ & \\
\hline Age $(20-89)^{\mathrm{b}}$ & & & & 0.077 & & & & 0.332 \\
\hline$<63$ & 50 & $26(52)$ & $24(48)$ & & 37 & $24(65)$ & $13(35)$ & \\
\hline$\geq 63$ & 49 & $34(69)$ & $15(31)$ & & 40 & $30(75)$ & $10(25)$ & \\
\hline Sex & & & & 0.769 & & & & 0.813 \\
\hline Male & 72 & $43(60)$ & $29(40)$ & & 55 & $39(71)$ & $16(29)$ & \\
\hline Female & 27 & $17(63)$ & $10(37)$ & & 22 & $15(68)$ & $7(32)$ & \\
\hline pT & & & & $0.004^{\mathrm{a}}$ & & & & 0.109 \\
\hline pT1 & 29 & $24(83)$ & $5(17)$ & & 25 & $21(84)$ & $4(16)$ & \\
\hline$\geq \mathrm{pT} 2$ & 70 & $36(51)$ & $34(49)$ & & 52 & $33(63)$ & $19(37)$ & \\
\hline pDiameter $(7-53 \mathrm{~mm})^{\mathrm{b}}$ & & & & $0.012^{\mathrm{a}}$ & & & & 0.076 \\
\hline$\leq 20 \mathrm{~mm}^{\mathrm{c}}$ & 51 & $37(73)$ & $14(27)$ & & 42 & $33(79)$ & $9(21)$ & \\
\hline$>20 \mathrm{~mm}$ & 48 & $23(48)$ & $25(52)$ & & 35 & $21(60)$ & $14(40)$ & \\
\hline DOI $(0-25 \mathrm{~mm})^{\mathrm{b}}$ & & & & $0.024^{\mathrm{a}}$ & & & & 0.467 \\
\hline$\leq 5 \mathrm{~mm}^{\mathrm{c}}$ & 39 & $29(74)$ & $10(26)$ & & 35 & $26(74)$ & $9(26)$ & \\
\hline$>5 \mathrm{~mm}$ & 60 & $31(52)$ & $29(48)$ & & 42 & $28(67)$ & $14(33)$ & \\
\hline Differentiation & & & & 0.170 & & & & 0.113 \\
\hline Well & 78 & $50(64)$ & $28(36)$ & & 62 & $46(74)$ & $16(26)$ & \\
\hline Mod and Por & 21 & $10(48)$ & $11(52)$ & & 15 & $8(53)$ & $7(47)$ & \\
\hline ly & & & & $0.001^{\mathrm{a}}$ & & & & $0.008^{\mathrm{a}}$ \\
\hline$(-)$ & 87 & $58(67)$ & $29(33)$ & & 69 & $52(75)$ & $17(25)$ & \\
\hline$(+)$ & 12 & $2(17)$ & $10(83)$ & & 8 & $2(25)$ & $6(75)$ & \\
\hline $\mathrm{v}$ & & & & $0.001^{\mathrm{a}}$ & & & & 0.055 \\
\hline$(-)$ & 67 & $48(72)$ & $19(28)$ & & 58 & $44(76)$ & $14(24)$ & \\
\hline$(+)$ & 32 & $12(38)$ & $20(63)$ & & 19 & $10(53)$ & $9(47)$ & \\
\hline neu & & & & 0.072 & & & & 0.117 \\
\hline$(-)$ & 82 & $53(65)$ & $29(35)$ & & 68 & $50(74)$ & $18(26)$ & \\
\hline$(+)$ & 17 & $7(41)$ & $10(59)$ & & 9 & $4(44)$ & $5(56)$ & \\
\hline TBG & & & & $<0.001^{\mathrm{a}}$ & & & & \\
\hline $1+2$ & 77 & $54(70)$ & $23(30)$ & & & & & \\
\hline 3 & 22 & $6(27)$ & $16(73)$ & & & & & \\
\hline PDPN & & & & $0.007^{\mathrm{a}}$ & & & & $0.002^{\mathrm{a}}$ \\
\hline$(-)$ & 39 & $30(77)$ & $9(23)$ & & 34 & $30(88)$ & $4(12)$ & \\
\hline$(+)$ & 60 & $30(50)$ & $30(50)$ & & 43 & $24(56)$ & $19(44)$ & \\
\hline \multicolumn{9}{|l|}{ Status } \\
\hline NED & 82 & $56(68)$ & $26(32)$ & & 68 & $52(76)$ & $16(24)$ & \\
\hline DOD & 17 & $4(24)$ & $13(76)$ & & 9 & $2(22)$ & $7(78)$ & \\
\hline
\end{tabular}

${ }^{\mathrm{a} P}<0.05$; ${ }^{\text {Range; }}{ }^{\mathrm{C}}$ Divided into two groups based on the UICC TNM eighth revised edition, a Fisher exact test or chi-square test. NLM, neck lymph node metastasis; pDiameter, pathological diameter; DOI, depth of invasion; Mod and Por, moderately and poorly; ly, lymphatic invasion; v, venous invasion; neu, perineural invasion; TBG, tumor budding grade; PDPN, podoplanin; NED, no evidence disease (including dead of another disease); DOD, dead of disease. Low TBG means TBG1 and 2.

found to be significant in all the patients (Table I). However, in the low TBG group, ly $(\mathrm{P}=0.008)$ and podoplanin $(\mathrm{P}=0.002)$ were significant for NLM.
The multivariate analysis of NLM was performed by using a logistic regression model with pDiameter, DOI and ly, TBG, and podoplanin ( $\mathrm{T}$ factor itself was not included 
TTable II. Multivariate analysis of NLM risk.

\begin{tabular}{|c|c|c|c|c|c|c|c|}
\hline \multirow[b]{2}{*}{ Characteristics } & \multirow[b]{2}{*}{ Value } & \multicolumn{3}{|c|}{ All cases $(n=99)$} & \multicolumn{3}{|c|}{ Low TBG cases $(n=77)$} \\
\hline & & OR & $95 \% \mathrm{CI}$ & P-value & OR & $95 \% \mathrm{CI}$ & P-value \\
\hline DOI & $\leq 5$ vs. $>5 \mathrm{~mm}^{\mathrm{a}}$ & 1.27 & $0.41-3.89$ & 0.678 & 0.88 & $0.26-3.01$ & 0.834 \\
\hline pDiameter & $\leq 20$ vs. $>20 \mathrm{~mm}^{\mathrm{a}}$ & 2.63 & $0.94-7.40$ & 0.067 & 1.97 & $0.61-6.37$ & 0.257 \\
\hline ly & $(-)$ vs. (+) & 9.78 & $1.68-56.8$ & $0.011^{\mathrm{b}}$ & 11.5 & $1.50-87.6$ & $0.019^{\mathrm{b}}$ \\
\hline TBG & $1+2$ vs. 3 & 4.71 & $1.47-15.1$ & $0.009^{\mathrm{b}}$ & & & \\
\hline PDPN & $(-)$ vs. (+) & 3.47 & $1.18-10.2$ & $0.023^{\mathrm{b}}$ & 7.07 & $1.80-27.7$ & $0.005^{\mathrm{b}}$ \\
\hline
\end{tabular}

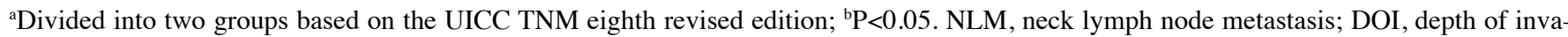
sion; pDiameter, pathological diameter; ly, lymphatic vessel invasion; TBG, tumor budding grade; PDPN, podoplanin; OR, odds ratio; CI, confidence interval. Low TBG means TBG 1 and 2.
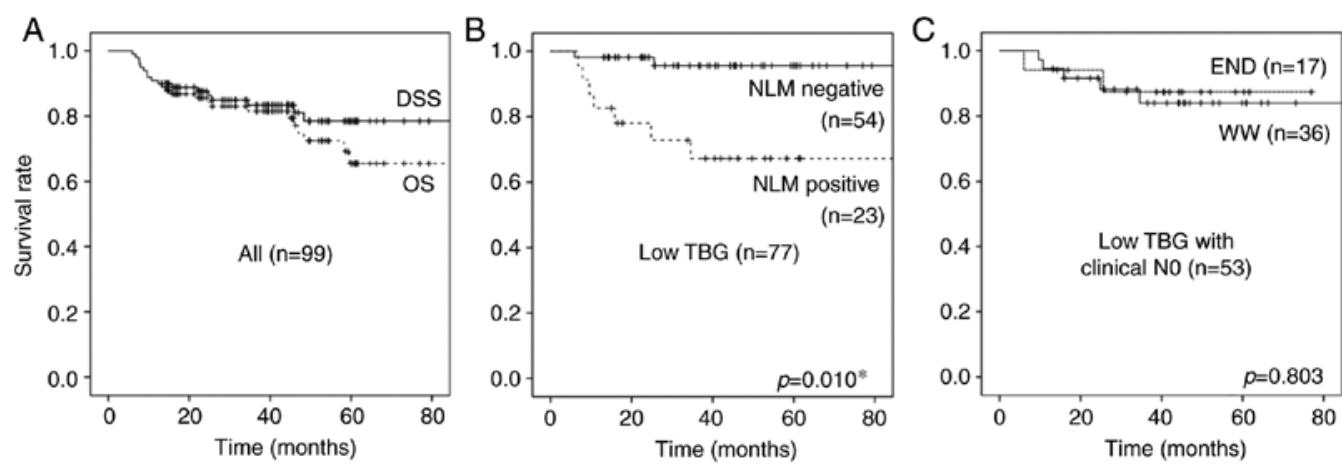

Figure 3. Survival curves and log-rank tests. (A) Survival curve of OS and DSS. (B) DSS of low TBG patients according to NLM. (C) DSS of low TBG with clinical N0 patients according to neck treatment strategy. $\mathrm{P}<0.05$ was considered to indicate a statistically significant difference. OS, overall survival; DSS, disease-specific survival; TBG, tumor budding grade; NLM, neck lymph node metastasis; WW, wait and watch policy; END, elective neck dissection.
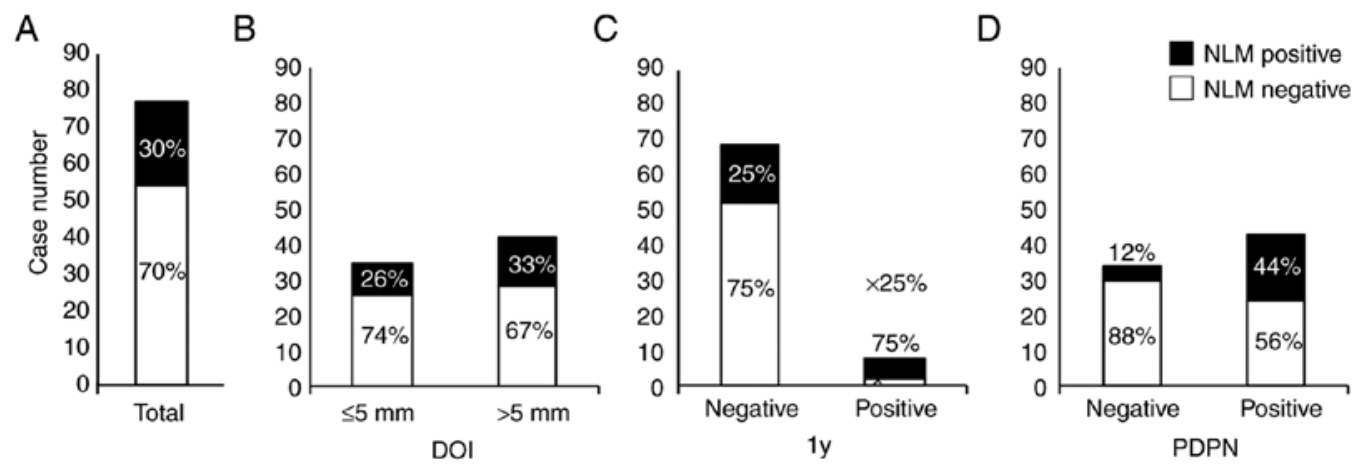

Figure 4. Number and ratio of NLM positive (black bar) and negative patients (white bar). (A) Total in the low TBG cases. (B) DOI. (C) ly. (D) PDPN. NLM, neck lymph node metastasis; TBG, tumor budding grade; DOI, depth of invasion; ly, lymphatic invasion; PDPN, podoplanin.

since pDiameter and DOI were components of $\mathrm{T}$ factor). The results showed that ly [odds ratio $(\mathrm{OR})=11.5,95 \%$ confidence interval (CI): 1.50-87.6; $\mathrm{P}=0.02$ ] and podoplanin $(\mathrm{OR}=7.07$, 95\% CI: $1.80-27.7 ; \mathrm{P}=0.005)$ were independent predictors of NLM (Table II). In the low TBG group, ly $(\mathrm{P}=0.019)$ and podoplanin $(\mathrm{P}=0.005)$ presented a significant difference.

We analyzed the predictive value of NLM. The ratio of NLM was $30 \%$ in the low TBG group (Fig. 4A). DOI was not significant for stratification of NLM (Fig. 4B). Lymphatic vessel invasion had a negative predictive value (NPV) of
$75 \%$ and positive predictive value (PPV) of $75 \%$ (Fig. 4C). Podoplanin had NPV of $88 \%$ and PPV of $44 \%$ (Fig. 4D).

\section{Discussion}

In this study, podoplanin expression was found to serve as a malignant index in the low TBG tongue SCC patients. In these patients, DOI was considered less useful, whereas ly and podoplanin were expected to become independent predictors of NLM. 
For patients with the low TBG, because of the high PPV, neck lymph node dissection is recommended if they are positive for ly. Lymphatic angiogenesis and expansion within a tumor is considered to promote tumor invasion and metastasis, based on the presumed passive metastasis mechanism (24). However, positivity for ly was only found in eight cases $(10 \%$ in low TBG cases), and 74\% of NLM-positive cases in the low TBG cases were negative for ly. Hence, in treating ly-negative patients, it may not be practical to adopt a wait and watch policy without neck lymph node dissection.

Moreover, the NPV of podoplanin (88\%) was superior to its PPV (56\%). Only four (12\%) podoplanin-negative cases were found to be NLM-positive, implying that podoplanin, compared to ly, has the advantage of fractionation in number. For podoplanin-negative in the low TBG patients, the wait and watch policy should be considered. This policy could reduce unnecessary neck dissections. Where high TBG can be distinguished by morphological appearance, malignant risks of low TBG cases can be distinguished by the combined use of immunohistochemical podoplanin expression. Neck dissection poses risks of complications and sequelae. It also causes prolonging of operation time or hospitalization period, in addition to cosmetic or functional problems, thus affecting patients' quality of life. Hence, selecting patients not requiring neck dissection is beneficial.

In our pilot study, we evaluated the degree of inflammation surrounding the buddings and analyzed its correlation with podoplanin expression using the Chi-squared test, resulting in a significant difference $(\mathrm{P}<0.05$, data not shown). This result is consistent with previous reports that inflammation is associated with podoplanin expression (16). In addition, podoplanin expression is thought to involve not only inflammation but also multiple factors like EMT. It is known that downregulation of E-cadherin and upregulation of vimentin occur when EMT is developed (25). Immunohistochemical staining of E-cadherin and vimentin was also conducted in our pilot study (data not shown). However, quantitative immunohistochemical evaluation of staining intensity, which means "increase and decrease" in these markers, is easily affected by various conditions, such as fixation and thickness of the section, which make reproducible and objective staining evaluation difficult. Moreover, vimentin is expressed on various non-epithelial cells, especially mesenchymal cells, including fibroblasts, endothelial cells, lymphocytes, and macrophages. This raises the difficulty in evaluating vimentin expression in buddings because of the positive reactions for vimentin in various cells. However, podoplanin showed the following advantages: it was expressed in fewer cells, and it was already widely established as a marker for detecting lymphatic invasion of tumor cells. Moreover, lymphovascular invasion was evaluated simultaneously when podoplanin expression in buddings was examined.

The limitations of this study were the small sample size and the lack of understanding of the mechanism underlying podoplanin expression in tumor buddings. Future research should expand the sample size to strengthen the relevance of the data presented in this study. The detailed mechanism of podoplanin expression in tumor budding cells has also yet to be elucidated. However, it is reported that podoplanin is affected by various phenomena, such as inflammation and wound healing, and is known to be associated with immune cells, cytokines, and EMT or non-EMT pathways $(16,19)$. Future comprehensive analyses, including investigation of variable podoplanin-related factors and standardization of the evaluation procedure, are expected to make podoplanin more useful.

In conclusion, the results of the present study emphasized that risk stratification by podoplanin expression associated with low TBG among patients with tongue SCC could provide a beneficial outcome. It could be expected to find the low TBG cases having a high NLM risk, based on the podoplanin-positive reaction. In general, these cases are difficult to predict the risk only morphologically. When the patients have not had a neck dissection, the podoplanin expression would be helpful for consideration of an additional treatment plan. This approach may be proposed as an accurate treatment algorithm for tongue SCC patients.

\section{Acknowledgements}

The authors would like to thank Mr. Kouichi Kamada and Ms. Akemi Miyata (Department of Pathology, Saitama Medical University International Medical Center) for their technical support.

\section{Funding}

The current study was supported by the Hidaka Research Projects from the Saitama Medical University (grant no. 31-D-1-11) and the Japan Society for the Promotion of Science KAKENHI Grant-in-Aid for Scientific Research C (grant no. 17K11404).

\section{Availability of data and materials}

The datasets used and/or analyzed during the current study are available from the corresponding author on reasonable request.

\section{Authors' contributions}

MH took part in conception, design, acquisition, analysis and interpretation of data, and drafted the manuscript. MYasuda took part in conception, design, critical revision of the manuscript for important intellectual content and supervision of the study. YE took part in conception, design, acquisition and drafted the manuscript. KN and MYano took part in analysis and interpretation of data and drafted the manuscript. YK took part in acquisition of data and important intellectual content. $\mathrm{MN}, \mathrm{MS}$ and HN took part in acquisition of data and supervision.

\section{Ethics approval and consent to participate}

The current study research was a retrospective study with approval of the Institutional Review Board at the Saitama Medical University International Medical Center (approval no. 17-201) and the contents of this study are open to the public at our hospital. All methods were performed in accordance with the 1975 Declaration of Helsinki. 


\section{Patient consent for publication}

Not applicable.

\section{Competing interests}

The authors declare that they have no competing interests.

\section{References}

1. NationalComprehensive CancerNetwork: NCCNClinical Practice Guidelines in Oncology: Head and Neck Cancers. Version 1.2019. https://www.ncen.org/store/login/login.aspx?ReturnURL=https:// www.nccn.org/professionals/physician_gls/pdf/head-and-neck. pdf. Accessed March 6, 2019.

2. Monroe MM and Gross ND: Evidence-based practice: Management of the clinical node-negative neck in early-stage oral cavity squamous cell carcinoma. Otolaryngol Clin North Am 45: 1181-1193, 2012.

3. Huang SH, Hwang D, Lockwood G, Goldstein DP and O'Sullivan B: Predictive value of tumor thickness for cervical lymph-node involvement in squamous cell carcinoma of the oral cavity: A meta-analysis of reported studies. Cancer 115: $1489-1497,2009$

4. Massano J, Regateiro FS, Januário G and Ferreira A: Oral squamous cell carcinoma: Review of prognostic and predictive factors. Oral Surg Oral Med Oral Pathol Oral Radiol Endod 102: 67-76, 2006.

5. Mitani S, Tomioka T, Hayashi R, Ugumori T, Hato N and Fujii S: Anatomic Invasive Depth Predicts Delayed Cervical Lymph Node Metastasis of Tongue Squamous Cell Carcinoma. Am J Surg Pathol 40: 934-942, 2016.

6. Kane SV, Gupta M, Kakade AC and D' Cruz A: Depth of invasion is the most significant histological predictor of subclinical cervical lymph node metastasis in early squamous carcinomas of the oral cavity. Eur J Surg Oncol 32: 795-803, 2006.

7. Adel M, Kao HK, Hsu CL, Huang JJ, Lee LY, Huang Y, Browne T, Tsang NM, Chang YL and Chang KP: Evaluation of Lymphatic and Vascular Invasion in Relation to Clinicopathological Factors and Treatment Outcome in Oral Cavity Squamous Cel Carcinoma. Medicine (Baltimore) 94: e1510, 2015.

8. Binmadi NO and Basile JR: Perineural invasion in oral squamous cell carcinoma: A discussion of significance and review of the literature. Oral Oncol 47: 1005-1010, 2011.

9. Brandwein-Gensler M, Teixeira MS, Lewis CM, Lee B, Rolnitzky L, Hille JJ, Genden E, Urken ML and Wang BY: Oral squamous cell carcinoma: Histologic risk assessment, but not margin status, is strongly predictive of local disease-free and overall survival. Am J Surg Pathol 29: 167-178, 2005.

10. Yamamoto E, Kohama G, Sunakawa H, Iwai M and Hiratsuka $H$ : Mode of invasion, bleomycin sensitivity, and clinical course in squamous cell carcinoma of the oral cavity. Cancer 51: 2175-2180, 1983.

11. Brierley JD, Gospodarowicz MK and Wittekind C (eds): UICC TNM Classification of Malignant Tumours. 8th edition. Wiley Blackwell, New York, NY, 2017.
12. Watanabe T, Muro K, Ajioka Y, Hashiguchi Y, Ito Y, Saito Y, Hamaguchi T, Ishida H, Ishiguro M, Ishihara S, et al; Japanese Society for Cancer of the Colon and Rectum: Japanese Society for Cancer of the Colon and Rectum (JSCCR) guidelines 2016 for the treatment of colorectal cancer. Int J Clin Oncol 23: 1-34, 2018.

13. Almangush A, Pirinen M, Heikkinen I, Mäkitie AA, Salo T and Leivo I: Tumour budding in oral squamous cell carcinoma: A meta-analysis. Br J Cancer 118: 577-586, 2018.

14. Zhu Y, Liu H, Xie N, Liu X, Huang H, Wang C and Hou J: Impact of tumor budding in head and neck squamous cell carcinoma: A meta-analysis. Head Neck 41: 542-550, 2019.

15. Ebihara Y, Yoshida S, Nakahira M, Kogashiwa Y, Enoki Y, Kuba K, Inoue H, Minami K, Yasuda $M$ and Sugasawa $M$ : Importance of tumor budding grade as independent prognostic factor for early tongue squamous cell carcinoma. Head Neck 41: 1809-1815, 2019.

16. Quintanilla M,Montero-Montero L, Renart J and Martín-Villar E: Podoplanin in Inflammation and Cancer. Int J Mol Sci 20: E707, 2019.

17. Puram SV, Parikh AS and Tirosh I: Single cell RNA-seq highlights a role for a partial EMT in head and neck cancer. Mol Cell Oncol 5: e1448244, 2018.

18. Puram SV, Tirosh I, Parikh AS, Patel AP, Yizhak K, Gillespie S, Rodman C, Luo CL, Mroz EA, Emerick KS, et al: Single-Cell Transcriptomic Analysis of Primary and Metastatic Tumor Ecosystems in Head and Neck Cancer. Cell 171: 1611-1624.e24, 2017.

19. Swain N, Kumar SV, Routray S, Pathak J and Patel S: Podoplanin - a novel marker in oral carcinogenesis. Tumour Biol 35: 8407-8413, 2014

20. Sobin LH, Gospodarowicz MK and Wittekind C (eds): UICC TNM Classification of Malignant Tumours. 7th edition. Wiley, West Sussex, 2009.

21. Lugli A, Kirsch R, Ajioka Y, Bosman F, Cathomas G, Dawson H, El Zimaity H, Fléjou JF, Hansen TP, Hartmann A, et al: Recommendations for reporting tumor budding in colorectal cancer based on the International Tumor Budding Consensus Conference (ITBCC) 2016. Mod Pathol 30: 1299-1311, 2017.

22. Xie N, Yu P, Liu H, Liu X, Hou J, Chen X, Huang H and Wang C: Validation of the International Tumor Budding Consensus Conference (2016) recommendations in oral tongue squamous cell carcinoma. J Oral Pathol Med 48: 451-458, 2019.

23. Kai K, Aishima S, Aoki S, Takase Y, Uchihashi K, Masuda M, Nishijima-Matsunobu A, Yamamoto M, Ide K, Nakayama A, et al: Cytokeratin immunohistochemistry improves interobserver variability between unskilled pathologists in the evaluation of tumor budding in T1 colorectal cancer. Pathol Int 66: 75-82, 2016.

24. Stacker SA, Williams SP, Karnezis T, Shayan R, Fox SB and Achen MG: Lymphangiogenesis and lymphatic vessel remodelling in cancer. Nat Rev Cancer 14: 159-172, 2014.

25. Aiello NM and Kang Y: Context-dependent EMT programs in cancer metastasis. J Exp Med 216: 1016-1026, 2019.

This work is licensed under a Creative Commons Attribution-NonCommercial-NoDerivatives 4.0 International (CC BY-NC-ND 4.0) License. 